Review

\title{
Chewing Maintains Hippocampus-Dependent Cognitive Function
}

\author{
Huayue Chen ${ }^{1 凶}$, Mitsuo Iinuma², Minoru Onozuka3 ${ }^{3}$ Kin-Ya Kubo ${ }^{\circledR}$ \\ 1. Department of Anatomy Gifu University Graduate School of Medicine, Gifu, 501-1194, Gifu, Japan \\ 2. Department of Pediatric Dentistry, Division of Oral Structure, Function and Development, Asahi University, School of Dentistry, Mi- \\ zuho, 501-0296, Gifu, Japan \\ 3. Department of Judo Therapy and Medical Science, Faculty of Medical Science, Nippon Sport Science University, Yokohama 227-0033, \\ Kanagawa, Japan \\ 4. Seijoh University Graduate School of Health Care Studies, Tokai, 476-8588, Aichi, Japan
}

$\triangle$ Corresponding author: Dr. H. Chen, Department of Anatomy Gifu University Graduate School of Medicine, 1-1 Yanagido, Gifu, 501-1194, Gifu, Japan. Phone: +81 58230 6295; Fax: +81 58230 6298; Email: huayue@gifu-u.ac.jp. Dr. Kin-ya Kubo, Seijoh University Graduate School of Health Care Studies, Tokai, 476-8588, Aichi, Japan. Email: kubo@seijoh-u.ac.jp

C 2015 Ivyspring International Publisher. Reproduction is permitted for personal, noncommercial use, provided that the article is in whole, unmodified, and properly cited. See http://ivyspring.com/terms for terms and conditions.

Received: 2015.02.16; Accepted: 2015.05.25; Published: 2015.06.09

\begin{abstract}
Mastication (chewing) is important not only for food intake, but also for preserving and promoting the general health. Recent studies have showed that mastication helps to maintain cognitive functions in the hippocampus, a central nervous system region vital for spatial memory and learning. The purpose of this paper is to review the recent progress of the association between mastication and the hippocampus-dependent cognitive function. There are multiple neural circuits connecting the masticatory organs and the hippocampus. Both animal and human studies indicated that cognitive functioning is influenced by mastication. Masticatory dysfunction is associated with the hippocampal morphological impairments and the hippocampus-dependent spatial memory deficits, especially in elderly. Mastication is an effective behavior for maintaining the hippocampus-dependent cognitive performance, which deteriorates with aging. Therefore, chewing may represent a useful approach in preserving and promoting the hippocampus-dependent cognitive function in older people. We also discussed several possible mechanisms involved in the interaction between mastication and the hippocampal neurogenesis and the future directions for this unique fascinating research.
\end{abstract}

Key words: Cognition, Hippocampus, Mastication

\section{Introduction}

With the rapid increase in the elderly populations, dementia has become an increasingly important health and socioeconomic issues [1]. Cognitive deficits are associated with aging as well as neurobiological, psychological and social factors [2,3]. Recently, attention has focused on the oral health conditions, especially mastication, as well as on the number of teeth, in the context of cognitive deficits. Both animal and human studies suggested a possible causal relationship between mastication and cognitive function [4-6]. Many older people have masticatory problems due to tooth loss, which compromises general health status. Some foods become difficult to eat for older people, most notably when tooth loss is present [7]. Loss of masticatory function is also associated with increased disability and mortality [8]. Consequently, being able to chew properly is of utmost important for elderly to maintain a healthy diet and preserve cognitive function. It has been demonstrated that mastication is of great importance for peripheral sensory input to the hippocampus for preserving and promoting the cognitive function. The systemic effect of the masticatory dysfunction is suggested to be an epidemiologic risk factor for dementia $[9,10]$. The importance 
of the cognitive modulation by the hippocampus is now widely recognized. Hippocampus is important for the formation and retrieval of episodic memories in humans [11]. Accumulating evidence indicates that impaired masticatory function causes morphological and functional alterations of the hippocampus [12-20]. Mastication or chewing helps to maintain the hippocampal function [20-23]. In the present manuscript, we aim to provide a comprehensive review, and a critical analysis of the current understanding of the relationship between mastication and the hippocampal functions.

\section{Functional analysis of masticatory appa- ratus}

The major masticatory components are bones, muscles, teeth, and soft tissues. Bones involved in mastication are maxilla and mandible. Mastication movements are executed using muscles connected to the maxilla and mandible. The mandible and maxilla are the anchor points for the deciduous or primary teeth in children and permanent teeth in adults. The soft tissues, including tongue, lips and cheeks, are also important in the manipulation of food during oral processing [24]. Mastication is the first stage of the digestion and involves the intermittent rhythmic act in which the tongue, facial and jaw muscles act in coordination to position the food between the teeth, cut it up and prepare it for swallowing. Mastication aims to decrease particle-size distribution in the food bolus and forms a cohesive bolus with the saliva in order to facilitate swallowing [24]. Teeth provide a uniquely discriminating sense of touch and directional specificity for occlusal awareness, intra-oral contact for management of a food bolus, discrimination of food texture and hardness, and control of jaw muscles for mastication and swallowing [25]. These specific features of teeth are closely linked with periodontal sensitivity suggesting an integrated role of the pulp-dentine-enamel complex [26]. These data further define the importance of teeth in the sensorimotor control of jaw function. The neural basis of the pulpal sensitivity contributes to the exquisite sensory discrimination mechanism of teeth. Somatosensory information from orofacial mechanoreceptors, such as periodontal, mucosal, muscle spindle, thermoreceptor, and gustatory and olfactory receptor, plays a critical role in the generation and control of jaw movements during mastication [25-27].

There are several methods to study mastication in animals and humans. In animal experiments, molar extraction $[13,14]$, occlusal disharmony or bite-raised condition [16, 17], soft-diet feeding [28, 29] and chewing wooden sticks $[21,22]$ are common used for examining masticatory functions. In human studies, the independent variables include self-reported dental status and chewing difficulty $[9,30]$. The oral cavity examination is a standardized protocol with regard to the number of natural teeth, occlusion and periodontal conditions.

\section{The neural circuits between masticatory organs and hippocampus}

A series of studies have demonstrated the contributions of various cerebral cortical areas for mastication [31]. Epidemiological research showed that loss of residual teeth, inadequate prostheses and a decrease in biting force are directly associated with the worsening of dementia $[9,30]$. In animal studies, mastication increases cerebral cortical blood flow and widely activates various cortical areas of the somatosensory, supplementary motor and insular cortices [32]. Blood oxygen levels in the prefrontal cortex and the hippocampus are increased by mastication, which may be essential for learning and memory processes [32]. Masticatory stimulation may prevent degradation of senile brain function and stress-related disorders without medication. The decrease of masticatory function, by either extraction of or reduction in molars and associated long-term soft-diet feeding, can inhibit learning and memory [12, 13, 16, 18, 19, 22, 33, 34]. Learning ability was shown to decrease in adult rats from 2 to 30 months after the extraction of molars [13, 28 , 29]. Soft-diet feeding also suppresses spatial learning ability from 6 to 12 months [28, 29, 35]. Spatial learning deteriorates after a period without molars longer than 2 months in young adult animals [33, 34]. Therefore, it is important to understand the relationship between mastication and brain function with aging.

The sensory information from the oral cavity including teeth is transmitted through the trigeminal sensory nerve to the trigeminal sensory nuclei, cerebellum, hypoglossal motor nuclei and the brainstem reticular formation [27, 31, 36, 37]. The reticular formation and the ascending reticular activating system are necessary for arousal of the brain for attention, perception and conscious learning. The neurons of the trigeminal sensory nuclei reach to the ventral posterior thalamic nucleus, the reticular formation and hypothalamus. The sensory information from the ventral posterior thalamus nucleus terminates on the somatosensory cortex. The neurons of the somatosensory cortex project their axons to the somatosensory association area, which has reciprocal projection with the entorhinal cortex. The entorhinal cortex is a major afferent source to the hippocampal dentate gyrus (DG). Therefore, the sensory information from the masticatory organs may affect hippocampus via thalamus and cerebral cortex. Hypo- 
thalamus receives input from the reticular formation and projects to hippocampus directly as opioidergic and histaminergic fibers. Hippocampus plays a vital role in controlling the release of various hormones by the hypothalamic-pituitary-adrenal (HPA) axis. Corticotrophin-releasing hormone $(\mathrm{CRH})$ produced by the hypothalamus stimulates the secretion of adrenocorticotropic hormone (ACTH) from the anterior pituitary, which stimulates the release of corticosterone from the adrenal cortex $[6,38]$. Due to the lipophilic nature, corticosterone can achieve rapid access to the brain. Hippocampus shows the highest density of glucocorticoid receptors (GR), making it a target for stress hormone actions [39]. Hippocampus receives projections of noradrenergic, serotonergic and dopaminergic fibers from the locus coeruleus, raphe nuclei and the ventral tegmental area, which are a part of the ascending reticular activating system. Thus, it is possible that mastication can influence the hippocampal function through the reticular formation. Therefore the effects of mastication on hippocampus may be attributable to multiple neural pathways.

\section{Hippocampal functional morphological changes caused the masticatory dysfunc- tion}

Evidence from neuroimaging studies suggests that masticatory dysfunction induced spatial memory and learning deficits associated with structural and functional reorganization of the hippocampus. Previous studies have elucidated the possible link between masticatory dysfunction and the hippocampus-dependent cognition using animal models, such as molar teeth extraction, crown reduction or bite elevation [14-18]. These animal models are able to chew, but the occlusal function markedly decreased, causing the degenerative changes in the periodontal mechanoreceptors, suggesting a suppression of sensory stimulation from the periodontal ligaments during chewing. In these experiments, molarless or bite-raised animals showed spatial memory deficits with the hippocampal morphological alterations [17].

\section{Hippocampal pathological morphology and masticatory dysfunction}

Animal studies have found that aged mice or rats with memory impairments have higher activity of the HPA axis [17-20]. HPA axis activity is negatively associated with both spatial memory performance and the hippocampal neurogenesis. Reduced masticatory stimulation decreased the hippocampal volume and induced the memory deficits. The pyramidal cell density of the hippocampal Cornu Ammonis 1 (CA1) and CA3 regions in the molarless or bite-raised mice was significantly decreased $[12,13,18]$. The number of dendritic spines reduced and the dendritic branching declined in an age-dependent manner [18, 20]. The dendritic spines in the hippocampus are altered by masticatory dysfunction through intracellular mechanisms that reorganize the neuronal cytoskeleton by producing cytoskeletal changes [40, 41]. Mice fed with a soft diet exhibited lower densities of synaptophysin- immunoreactive terminals and synaptic formation in the hippocampus [34].

Neurogenesis is well-established to occur in the subgranular zone (SGZ) of the hippocampal DG and the forebrain subventricular zone (SVZ) throughout life [42]. DG is typically considered to have a key role in the encoding of spatial and contextual information, particularly in pattern separation and novelty detection [43]. The newly generated neurons in the SGZ migrate to the inner granule cell layer, rapidly extend long axonal projections along the mossy fiber pathway and reach their target CA3 pyramidal neuronal cell layer, form connections with the CA3 neurons, hilar interneurons and release glutamate as their main neurotransmitter thus attaining a functional significance. Newly generated cells in the adult mouse hippocampus are found to exhibit neuronal morphology and display passive membrane properties, action potentials and functional synaptic inputs similar to those found in the mature DG cells. The rate of neurogenesis is modulated by various physiological and pathological conditions. The newly generated cells may have a function in cognition and brain repair mechanisms. Animal studies showed that prolonged masticatory dysfunction suppresses the neurogenesis of DG granule neurons [44]. The neurogenesis both in the SVZ and the hippocampal DG decreased in the soft-diet-fed mice [45]. Molarless or bite-raised condition in mice and rats significantly decreased cell proliferation in the hippocampal DG in an age-dependent manner [15, 21]. After extracting molar teeth or raising the bite in aged mice, the cell proliferation decreased abruptly, and then increased, but did not recover to the control levels. These findings suggest that reduced mastication impairs cell proliferation in the hippocampal DG, leading to spatial learning and memory deficits.

\section{Hippocampal molecular morphology and masticatory dysfunction}

The prototypical immediate early gene c-fos is transcribed in neurons within minutes after stimulation by various depolarizing and neurotrophic intercellular signals, and rapid degradation of c-fos mRNA ensures that its expression represents recent changes in neuronal activities [46]. Fos protein is an indicator of the neural plasticity and is strongly implicated in 
memory formation. Experience and spatial learning stimulate Fos expression. It was demonstrated that c-fos knockout mice exhibited deficits in long-term memory and synaptic plasticity [47]. Moreover, memory impairment following brain ischemia is often associated with decreased c-fos expression [48]. Masticatory dysfunction caused a reduction in the number of Fos-positive cells in CA1 region. This effect was more pronounced the longer the molarless condition persisted. The suppression of Fos induction in CA1 region induced by the molarless condition was reduced by restoring the lost molars with artificial crowns [49].

The cholinergic system of the basal forebrain is one of the key transmitter systems for learning and memory. Hippocampus receives abundant regulatory inputs from the basal forebrain cholinergic system. The hippocampal cholinergic system plays a crucial role in spatial learning [50]. Treatment with donepezil, a potent and selective acetylcholinesterase inhibitor, significantly enhanced the survival of newborn neurons. Donepezil treatment could reverse the chronic stress-induced decrease in neurogenesis. Activation of the cholinergic system promotes survival of newborn neurons in the adult DG under both normal and stressed conditions [51]. Administration with neurotoxin 192 IgG-saponin selectively destroyed cholinergic neurons of basal forebrain, impaired neurogenesis in the granule cell layers and increased apoptotic cell numbers specifically in the subgranular zone of DG region [52]. The pro-cognitive influences of the cholinergic system in the hippocampus involved the production of new neurons, which contribute to DG synaptic plasticity and memory trace formation. Targeting the cholinergic system with cholinesterase inhibitors, which leads to cognitive improvement in patients afflicted with age-related dementia, could possibly exert its therapeutic action through stimulation of hippocampal neurogenesis [51, 52]. In the molarless or bite-raised aged mice, hippocampal acetylcholine release, acetyltransferase and choline acetyltransferase activity were significantly decreased $[13,53]$. The number of choline acetyltransferase-immunopositive neurons in the medial septal nucleus was also reduced. However, the molarless or bite-raised condition had little effect in young mice, indicating an age-dependent decrease in the hippocampal cholinergic system. Reduced masticatory activity in mice may be involved in the development of age-related functional impairment of the hippocampal cholinergic system.

Recent studies showed that impairment of masticatory function downregulated memory-related signaling pathways and genes in the hippocampal neurons, including brain-derived neurotrophic factor
(BDNF) and its receptor, tropomyosin-related kinase $\mathrm{B}$ (trkB) $[54,55]$. BDNF is a family of nerve growth factors which bind to trkB. In response to BDNF signaling, its specific high-affinity receptor trkB has an enhancing effect on nerve transmission. Both BDNF and trkB have been shown to be important modulators of synaptic plasticity and are critical to the development of the central nervous system [56]. Animal experiments have demonstrated that BDNF is required for the maintenance of dendritic spines in the adult brain [57]. BDNF-TrkB signaling can influence dendritic spine and synapse density in hippocampal neurons [56]. The expression of trkB-mRNA has been used as an effective marker for elevated synaptic transmission levels in the signaling pathways related to spatial learning. The trkB-mRNA levels were significantly lower in tooth extraction rats $[56,57]$. The spatial memory and the number of trkB-mRNA-positive cells were both negatively affected by the duration of tooth loss and the number of teeth extracted. As a mediator of the hippocampal-dependent learning and memory, the BDNF-trkB binding plays a critical role in activity-dependent synaptic plasticity. The spatial memory impairment in rats could have a close relationship with the decrease in the trkB levels of the pathways located from the trigeminal nerve area to the hippocampus.

\section{Hippocampal astrocyte and masticatory dysfunction}

Astrocytes undergo modification, leading to hypertrophy and hyperplasia, increasing the production of pro-inflammatory cytokines, such as microglial-derived IL-1 $\beta$. The hypersecretion of immunomodulating cytokines and the consequent inflammatory responses in the astrocytes and neurons are frequently observed in aged hippocampus, attributed to age-related cognitive impairment [58]. Astrocytes play a central role virtually in all forms of neuropathology, determining, to a large extend, the progression and outcome of neurologic diseases [59]. Glial fibrillary acidic protein (GFAP) is considered to be a universal glial marker and is generally acknowledged as a sign of pathological response of astroglia, the reactive astrogliosis. GFAP-positive astrocytes vary between brain regions, with about $80 \%$ of cells in the hippocampus [60]. Aging is associated with astrocyte proliferation and increase in the expression of GFAP, both considered to be signs of reactive astroglial hypertrophy [61]. Molarless or bite-raised mice or rats showed hypertrophy of astrocytes in CA1, suggesting glial cells are inflamed and degenerating, implying that the production of cytokines is increased [4, 14]. The number of GFAP-positive astrocytes increased with aging, especially in molarless or bite-raised mice. 
Masticatory deficiency not only caused neuronal degeneration, but also induced an increase of astrocyte number accompanied by morphological and physiological changes of the hippocampus.

Sensory input from the masticatory organs is essential to maintain the hippocampus-dependent learning. Masticatory impairment of the young animals may not affect the hippocampus in the short term, as the hippocampus receives various peripheral sensory inputs continuously to maintain its function. In aged animals, however, reduced masticatory activity and degenerative periodontal mechanoreceptors do not provide sufficient sensory input to maintain the hippocampal function, leading to impairment of the hippocampal cognitive performance. For young mice, a period longer than two months is required to decrease the learning ability. Aged molarless or bite-raised mice rapidly developed the learning deficits $[4,6,14,19]$.

\section{Chewing stimulation helps to maintain the hippocampal function}

Several human studies have showed that chewing is effective for preserving hippocampal function, which deteriorates with aging $[9,62-66]$. Based on large-scale survey data from several European countries, Listl provided novel epidemiological evidence supportive of an association between oral health and cognitive functioning [67]. A population-based cross-sectional study suggested that natural teeth are important for the hippocampus-based cognitive processes, such as episodic long-term memory [30]. Chewing or even sucking a piece of sugar-free, spearmint flavoured gum was shown to be associated with immediate better cognitive performance, improved the score of word recall, sensitivity index of the spatial working-memory task, and reaction time of the numeric working-memory task in humans. Chewing increased the cerebral blood flow and might decrease the risk of cognitive impairments [62-64]. An association between chewing ability and cognition was found in a population-based study. Rural community residents aged 70-74 without dementia, having poor chewing ability were found to perform worse on the same cognitive tests [65]. In another study, the association between chewing gum and cognitive performance depended on individual differences such as feeling thirsty and being an introvert [66]. Lexomboon and colleagues conducted an interesting study concerning the relationship between chewing ability, tooth loss, and cognitive impairments in a general Swedish older population [9]. They found that the association between chewing ability and cognitive impairments was stronger than that between tooth loss and cognitive impairment when adjusted for age, sex, and education. They suggested that chewing itself may positively influence cerebral blood flow, alleviated stress, and therefore enhance cognitive ability. Recently, Brobeil et al. reported that protein tyrosine phosphatase interacting protein 51 (PTPIP51) is probably a new protein regulating memory formation by influencing dendritic and axonal growth and synaptogenesis [68]. The expression of PTPIP51 was specifically restricted to the cerebellum and the hippocampus [69], suggesting the interaction between cerebellum and hippocampus. Functional magnetic resonance imaging (fMRI) study revealed that chewing affects the cerebellar functions [62]. Cerebellum may be involved in chewing rhythmicity and motor control motivated by proprioceptive inputs. The increase in the cerebellar neural activity during chewing may improve the hippocampus-dependent memory and cognition.

In animal experiments, mice fed a soft diet showed low neurogenesis, while mice fed only a hard diet or a hard diet after a soft one showed normal or recovered neurogenesis [45]. This study suggests that feeding with a hard diet improves the neurogenesis. Chewing under restraint condition reversed the stress-induced suppression of cell proliferation in the DG [6, 21]. Chewing during the stressful condition attenuated the effect of stress on cognitive function. Chewing during a stress event altered the HPA axis function and improved the ability to cope with stress in rodents [21, 70-73]. Chewing during restrain stress significantly suppressed the stress-induced enhancement of $\mathrm{CRH}$ expression in the paraventricular nucleus [74]. The number of stress-suppressed GR-immunopositive neurons in the hippocampal CA1 region was increased after chewing stimulation [74]. GRs play an important role in memory consolidation and retrieval [75]. Neuroendocrine activation of the HPA axis is counteracted by GR-mediated negative feedback, which terminates the stress response. Masticatory dysfunction inhibited the negative-feedback response by downregulation of GR protein and mRNA expression [16]. In aged mice, the molarless or bite-raised condition suppressed cell proliferation in the hippocampal DG [15, 21]. Pretreatment with metyrapone, the corticosterone synthesis blocker, inhibited the increase in plasma corticosterone level induced by the bite-raised condition, and also attenuated the reduction in cell proliferation [21]. Administration of metyrapone attenuated the chronic stress-induced neuronal degeneration in the hippocampus and chronic stress-induced impairments in spatial learning in rats [76]. Immobilization stress suppressed cell proliferation in hippocampal DG region. However, chewing under restraint stress blocked the stress-induced suppression of cell prolif- 
eration in the DG region. The functional morphologic deficits induced by the molarless condition in aged SAMP8 mice was a result of increased plasma corticosterone levels, and that chewing under restraint stress prevented the stress-induced suppression of the neurogenesis in the DG, which is required for the hippocampus- dependent learning and memory in adults. Thus, chewing during stress event may attenuate stress-induced impairment in cognitive function.

Chewing during stress in rats and mice attenuated stress-induced increase in corticosterone level, CRH expression, c-Fos protein induction, phosphorylation of extracellular signal-related protein kinase $1 / 2$, oxidative stress and nitric oxide in the hypothalamus. Chewing also attenuates stress-induced impairments of plasticity in the hippocampus by activating stress-suppressed N-methyl-D-aspartate (NMDA) receptor-mediated long-term potentiation (LTP), which is responsible for learning and memory [71]. Stress caused prolonged activation of the HPA axis, stimulating adrenal cortex to secrete corticosterone. Increased corticosterone level caused a $\mathrm{Ca}^{2+}$ influx into neurons via activation of corticosterone receptors, attenuated cellular excitability and weakened hippocampal LTP selectively that depends on NMDA receptor [71, 77]. Stress-attenuated hippocampal plasticity could be counteracted when the rats have an opportunity to behaviorally respond to the stress by chewing a wooden stick. Chewing under stress ameliorated stress-induced hippocampal impairment by rescuing NMDAR function [71, 77]. Chewing also could induce histamine release and stimulate histamine $\mathrm{H} 1$ receptor activation in the hippocampus. Chewing-induced activation of the histaminergic nervous system in the hippocampus facilitated the extracellular histamine level and restored stress-attenuated NMDA receptor function in the hippocampus.

Chewing could reverse the HPA axis function, improve the ability to cope with stress and alleviate chronic stress-induced hippocampus-dependent spatial learning deficits. Effects of chewing on the hippocampal function may be attributable to the multiple pathways, including various regulatory nervous inputs, memory-related genes and signaling pathways, stress hormone and its receptors. It was reported that gum chewing improved the performance of memory recall in elderly subjects, but did not show any effects in the young adult subjects [4,78]. As compared with young animals, chewing is more effective on the aged animals by supplying additional sensory inputs to improve hippocampal function, which deteriorates with aging.

\section{Conclusions and the future directions}

Mastication plays an important role in preserving the hippocampus-dependent cognitive function. Masticatory deficiency impairs the hippocampal morphology via the neural circuits and HPA axis, resulting in spatial memory and learning deficits. Mastication during stress event could reverse stress-induced neuronal degeneration and suppression of cell proliferation in the hippocampus and attenuates stress-induced impairments of plasticity in the hippocampus by various regulatory nervous inputs, memory-related genes and signaling pathways, stress hormone and its receptors. Therefore, chewing might be an effective approach in maintaining the hippocampus-related spatial learning and memory. Chewing may represent a useful approach in preserving and promoting the hippocampus-dependent cognitive function in older people.

A full understanding of the mechanisms mediating mastication-induced hippocampal neurogenesis is complicated by the involvement of multiple regulatory inputs, including stress hormone and its receptor, neurotransmitter systems, memory-related genes and signaling pathways. Research to date has tended to focus on the contribution of the masticatory stimulation beneficial for hippocampal function. There is a paucity of information on how the multiplicity of substrates, neurotransmitter systems and genes interact with one another to modulate the interaction between mastication and hippocampal function. Future studies should focus on cross-talk between neurotransmitter and receptor systems and adopt a neural networks approach to better understand hippocampal function. There is no doubt that the numerous animal models that have been developed have facilitated an increased understanding the relationship between mastication and the hippocampal functional morphology. It may be worth considering which of these animal models most appropriately models in humans. This is a complex consideration and it is very difficult to single out any one model as being the one that most closely models the human condition when the human condition itself is not yet fully understood. There is a need for further studies examining the association between mastication and the hippocampus- dependent cognition over the life-course, and the influence of aging on cognition, in animal models and humans.

\section{Acknowledgements}

This work was supported by a Grant-in-aid for Scientific Research from the Japan Society for the Promotion of Science to H.C. (No. 26462916). 


\section{Competing Interests}

The authors have declared that no competing interest exists.

\section{References}

1. Reitz C, Brayne C, Mayeux R. Epidemiology of Alzheimer disease. Nat Rev Neurol. 2011; 7:137-152.

2. Gerstorf D, Herlitz A, Smith J. Stability of sex differences in cognition in advanced old age: the role of education and attrition. J Gerontol B Psychol Sci Soc Sci. 2006; 61: 245-249.

3. Habib R, Nyberg L, Nilsson LD. Cognitive and non-cognitive factors contributing to the longitudinal identification of successful older adults in the betula study. Neuropsychol Dev Cogn B Aging Neuropsychol Cogn. 2007; 14: 257-273.

4. Ono Y, Yamamoto T, Kubo KY, Onozuka M. Occlusion and brain function: mastication as a prevention of cognitive dysfunction. J Oral Rehabil. 2010; 37: 624-640.

5. Teixeira FB, Fernandes LM, Noronha PA, et al. Masticatory deficiency as a risk factor for cognitive dysfunction. Int J Med Sci. 2014; 11: 209-214.

6. Kubo KY, Chen H, Onozuka M. The relationship between mastication and cognition. In: Wang Z, Inuzuka $H$, eds. Senescence and senescence-related disorders. Rijeka: InTech; 2013: 115-132.

7. Locker D. Changes in chewing ability with ageing: a 7-year study of older adults. J Oral Rehabil. 2002; 29: 1021-1029.

8. Shimazaki $\mathrm{Y}$, Soh I, Saito T, et al. Influence of dentition status on physical disability, mental impairment, and mortality in institutionalized elderly people. J Dent Res. 2001; 80: 340-345.

9. Lexomboon D, Trulsson M, Wårdh I, Parker MG. Chewing ability and tooth loss: association with cognitive impairment in an elderly population study. J Am Geriatr Soc. 2012; 60: 1951-1956.

10. Stein PS, Desrosiers M, Donegan SJ, et al. Tooth loss, dementia and neuropathology in the Nun study. J Am Dent Assoc. 2007; 138: 1314-1322.

11. Henke K. A model for memory systems based on processing modes rather than consciousness. Nat Rev Neurosci. 2010; 11: 523-532.

12. Onozuka M, Watanabe K, Mirbod SM, et al. Reduced mastication stimulates impairment of spatial memory and degeneration of hippocampal neurons in aged SAMP8 mice. Brain Res. 1999; 826: 148-153.

13. Onozuka M, Watanabe K, Nagasaki S, et al. Impairment of spatial memory and changes in astroglial responsiveness following loss of molar teeth in aged SAMP8 mice. Behav Brain Res. 2000; 108: 145-155.

14. Onozuka M, Watanabe K, Fujita M, Tomida M, Ozono S. Changes in the septohippocampal cholinergic system following removal of molar teeth in the aged SAMP8 mouse. Behav Brain Res. 2002; 133: 197-204.

15. Watanabe K, Tonosaki K, Kawase T, et al. Evidence for involvement of dysfunctional teeth in the senile process in the hippocampus of SAMP8 mice. Exp Gerontol. 2001; 36: 283-295.

16. Mori D, Katayama T, Miyake H, Fujiwara S, Kubo KY. Occlusal disharmony leads to learning deficits associated with decreased cellular proliferation in the hippocampal dentate gyrus of SAMP8 mice. Neurosci Lett. 2013; 534: 228-232.

17. Ichihashi $Y$, Arakawa Y, Iinuma M, et al. Occlusal disharmony attenuates glucocorticoid negative feedback in aged SAMP8 mice. Neurosci Lett. 2007; 427: 71-76.

18. Kubo KY, Yamada $\mathrm{Y}$, Iinuma $\mathrm{M}$, et al. Occlusal disharmony induces spatial memory impairment and hippocampal neuron degeneration via stress in SAMP8 mice. Neurosci Lett. 2007; 414: 188-191.

19. Kawahata M, Ono Y, Ohno A, et al. Loss of molars early in life develops behavioral lateralization and impairs hippocampus-dependent recognition memory. BMC Neurosci. 2014; 15: 4.

20. Kubo KY, Iwaku F, Watanabe K, Fujita M, Onozuka M. Molarless-induced changes of spines in hippocampal region of SAMP8 mice. Brain Res. 2005; 1057: 191-195.

21. Kubo KY, Sasaguri K, Ono Y, et al. Chewing under restraint inhibits the stress-induced suppression of cell birth in the dentate gyrus of aged SAMP8 mice. Neurosci Lett. 2009; 466: 109-113.

22. Onishi M, Iinuma M, Tamura Y, Kubo KY. Learning deficits and suppression of the cell proliferation in the hippocampal dentate gyrus of offspring are attenuated by maternal chewing during prenatal stress. Neurosci Lett. 2014; 560: $77-80$.

23. Hirano $\mathrm{Y}$, Obata $\mathrm{T}$, Takahashi $\mathrm{H}$, et al. Effects of chewing on cognitive processing speed. Brain Cogn. 2013; 81: 376-381.

24. Trulsson M, van der Bilt A, Carlsson GE, et al. From brain to bridge: masticatory function and dental implants. J Oral Rehabil. 2012; 39: 858-877.

25. Klineberg IL, Trulsson M, Murray GM. Occlusion on implants-is there a problem? J Oral Rehabil. 2012; 39: 522-537.

26. Farahani RM, Simonian M, Hunter N. Blueprint of an ancestral neurosensory organ revealed in glial network in human dental pulp. J Comp Neurol. 2011; 519: 3306-3326.

27. Capra NF. Mechanisms of oral sensation. Dysphagia. 1995; 10: 235-247.

28. Yamamoto T, Hirayama A. Effects of soft-diet feeding on synaptic density in the hippocampus and parietal cortex of senescence-accelerated mice. Brain Res. 2001; 902: 255-263.
29. Aoki H, Kimoto K, Hori N, Toyoda M. Cell proliferation in the dentate gyrus of rat hippocampus is inhibited by soft diet feeding. Gerontology. 2005; 51: 369-374.

30. Hansson P, Sunnegårdh-Grönberg K, Bergdahl J, et al. Relationship between natural teeth and memory in a healthy elderly population. Eur J Oral Sci. 2013; 121: 333-340.

31. Takemura M, Sugiyo S, Moritani M, et al. Mechanisms of orofacial pain control in the central nervous system. Arch Histol Cytol. 2006; 69: 79-100.

32. Miyake S, Wada-Takahashi S, Honda H, et al. Stress and chewing affect blood flow and oxygen levels in the rat brain. Arch Oral Biol. 2012; 57: 1491-1497.

33. Kato T, Usami T, Noda Y, et al. The effect of the loss of molar teeth on spatial memory and acetylcholine release from the parietal cortex in aged rats. Behav Brain Res. 1997; 83: 239-342.

34. Yamazaki K, Wakabayashi N, Kobayashi T, Suzuki T. Effect of tooth loss on spatial memory and trkB-mRNA levels in rats. Hippocampus. 2008; 18: 542-547.

35. Tsutsui K, Kaku M, Motokawa M, et al. Influences of reduced masticatory sensory input from soft-diet feeding upon spatial memory/learning ability in mice. Biomed Res. 2007; 28: 1-7

36. Sessle BJ, Adachi K, Avivi-Arber L, et al. Neuroplasticity of face primary motor cortex control of orofacial movements. Arch Oral Biol. 2007; 52: 334-337.

37. Malick A, Burstein R. Cells of origin of the trigeminohypothalamic tract in the rat. J Comp Neurol. 1998; 400: 125-144.

38. Iinuma M, Kondo $\mathrm{H}$, Kurahashi $\mathrm{M}$, et al. Relationship between the early toothless condition and hippocampal functional morphology. Anatomy \& Physiology. 2014; 4: 3.

39. De Kloet ER, Vreugdenhil E, Oitzl MS, Joëls M. Brain corticosteroid receptor balance in health and disease. Endocr Rev. 1988; 19: 269-301.

40. Kulkarni VA, Firestein BL. The dendritic tree and brain disorders. Mol Cell Neurosci. 2012; 50: 10- 20.

41. Penzes P, Rafalovich I. Regulation of the actin cytoskeleton in dendritic spines. Adv Exp Med Biol. 2012; 970: 81-95.

42. Kuhn HG, Dickinson-Anson H, Gage FH. Neurogenesis in the dentate gyrus of the adult rat: Age- related decrease of neuronal progenitor proliferation. J Neurosci. 1996; 16: 2027-2033.

43. Kesner RP. An analysis of the dentate gyrus function. Behav Brain Res. 2013; 254: $1-7$.

44. Schoenfeld TJ, Gould E. Differential effects of stress and glucocorticoids on adult neurogenesis. Curr Top Behav Neurosci. 2013; 15: 139-164.

45. Utsugi C, Miyazono S, Osada K, et al. Hard-diet feeding recovers neurogenesis in the subventricular zone and olfactory functions of mice impaired by soft-diet feeding. PLos One 2014; 9: e97309.

46. Kovacs KJ. Measurement of immediate-early gene activation-c-fos and beyond. J Neuroendocrinol. 2008; 20: 665-672.

47. Guzowski JF, Setlow B, Wagner EK, McGaugh JL. Experience-dependent gene expression in the rat hippocampus after spatial learning: a comparison of the immediate-early genes Arc, c-fos, and zif268. J Neurosci. 2001; 21: 5089-5098.

48. Han X, Zhao X, Lu M, et al. Electroacupuncture ameliorates learning and memory via activation of the CREB signaling pathway in the hippocampus to attenuate apoptosis after cerebral hypoperfusion. Evid Based Complement Alternat Med. 2013; 2013: 156489.

49. Watanabe K, Ozono S, Nishiyama K, et al. The molarless condition in aged SAMP8 mice attenuates hippocampal Fos induction linked to water maze performance. Behav Brain Res. 2002; 128: 19-25.

50. Bartus RT, Dean RL 3rd, Beer B, Lippa AS. The cholinergic hypothesis of geriatric memory dysfunction. Science. 1982; 217: 408-414.

51. Kaneko N, Okano H, Sawamoto K. Role of the cholinergic system in regulating survival of newborn neurons in the adult mouse dentate gyrus and olfactory bulb. Genes Cells. 2006; 11: 1145-1159.

52. Mohapel $P$, Leanza G, Kokaia M, Lindvall O. Forebrain acetylcholine regulates adult hippocampal neurogenesis and learning. Neurobiol Aging. 2005; 26: 939-946.

53. Katayama T, Mori D, Miyake H, et al. Effect of bite-raised condition on the hippocampal cholinergic system of aged SAMP8 mice. Neurosci Lett. 2012; 520: 77-81.

54. Iida S, Hara T, Araki D, et al. Memory-related gene expression profile of the male rat hippocampus induced by teeth extraction and occlusal support recovery. Arch Oral Biol. 2014; 59: 133-141.

55. Monsey MS, Boyle LM, Zhang ML, et al. The expression of memory-related genes in the lateral amygdala and enhances the consolidation of a pavlovian fear memory. PLoS One. 2014; 9: e91530.

56. Cohen-Cory $\mathrm{S}$, Kidane $\mathrm{AH}$, Shirkey NJ, Marshak $\mathrm{S}$. Brain-derived neurotrophic factor and the development of structural neuronal connectivity. Dev Neurobiol. 2010; 70: 271-288.

57. Vigers AI, Amin DS, Talley-Farnham $\mathrm{T}$, et al. Sustained expression of brain-derived neurotrophic factor is required for maintenance of dendritic spines and normal behavior. Neuroscience. 2012; 212: 1-18.

58. Rodríguez JJ, Yeh CY, Terzieva S. Complex and region-specific changes in astroglial markers in the aging brain. Neurobiol Aging. 2014; 35: 15-23.

59. Parpura V, Heneka MT, Montana V, et al. Glial cells in (patho) physiology. J Neurochem. 2012; 121: 4-27.

60. Kimelberg HK. The problem of astrocyte identity. Neurochem Int. 2004; 45: 191-202.

61. Cotrina ML, Nedergaard M. Astrocytes in the aging brain. J Neurosci Res. 2002; 67: 1-10. 
62. Onozuka M, Fujita M, Watanabe $\mathrm{K}$, et al. Mapping brain region activity during chewing: a functional magnetic resonance imaging study. J Dent Res. 2002; 81: 743-746.

63. Hirano $\mathrm{Y}$, Obata $\mathrm{T}$, Takahashi $\mathrm{H}$, et al. Effects of chewing on cognitive processing speed. Brain Cogn. 2013; 81: 376-381.

64. Hasegawa Y, Tachibana Y, Sakagami J, et al. Flavor-Enhanced Modulation of Cerebral Blood Flow during Gum Chewing. PLoS One. 2013; 8: e66313.

65. Moriya S, Tei K, Murata A, et al. Associations between self-assessed masticatory ability and higher brain function among the elderly. J Oral Rehabil. 2011; 38: 746-753.

66. Stephens R, Edelstyn NMJ. Do individual differences moderate the cognitive benefits of chewing gum? Psychology. 2011; 2: 834-840.

67. Listl S. Oral health conditions and cognitive functioning in middle and later adulthood. BMC Oral Health. 2014; 14: 70.

68. Brobeil A, Viard M, Petri MK, et al. Memory and PTPIP51 - A new protein in hippocampus and cerebellum. Mol Cell Neurosci. 2015; 64: 61-73.

69. Koch P, Viard M, Stenzinger A, et al. Expression profile of PTPIP51 in mouse brain. J Comp Neurol. 2009; 517: 892-905.

70. Ono Y, Kataoka T, Miyake S, et al. Chewing ameliorates stress-induced suppression of hippocampal long-term potentiation. Neuroscience. 2008; 154: 1352-1359.

71. Ono Y, Kataoka T, Miyake S, et al. Chewing rescues stress-suppressed hippocampal long-term potentiation via activation of histamine H1 receptor. Neurosci Res. 2009; 64: 385-390.

72. Miyake S, Yoshikawa G, Yamada K, et al. Chewing ameliorates stress-induced suppression of spatial memory by increasing glucocorticoid receptor expression in the hippocampus. Brain Res. 2012; 1446: 34-39.

73. Kubo KY, Chen H. Mastication as a stress-coping behavior. Biomed Res Int. 2015; 2015: 876409 .

74. Hori N, Yuyama N, Tamura K. Biting suppresses stress-induced expression of corticotropin- releasing factor (CRF) in the rat hypothalamus. J Dent Res. 2004; 83: 124-128.

75. Oitzl MS, Rechardt HM, Joëls M, de Kloet ER. Point mutation in the mouse glucocorticoid receptor preventing DNA binding impairs spatial memory. Proc Natl Acad Sci USA. 2001; 98: 12790-12795

76. Wright RL, Lightner EN, Harman JS, Meijer OC, Conrad CD. Attenuating corticosterone levels on the day of memory assessment prevents chronic stress-induced impairments in spatial memory. Eur J Neurosci. 2006; 24: 595-606.

77. Wiegert O, Pu Z, Shor S, Joëls M, Krugers H. Glucocorticoid receptor activation selectively hampers $\mathrm{N}$-methyl-D-aspartate receptor dependent hippocampal synaptic plasticity in vitro. Neuroscience. 2005; 135: 403-411.

78. Onozuka M, Hirano $\mathrm{Y}$, Tachibana A, et al. Interactions between chewing and brain activities in humans. In: Onozuka M, Yen CT, eds. Novel trends in brain science. Tokyo: Springer; 2007: 99-113. 